\title{
Espécies nativas que podem substituir as exóticas no paisagismo(1)
}

\author{
ELISABETH REGINA TEMPEL STUMPF(2); PATRICK DA SILVA SILVA(2); \\ ISADORA DIAS ROMAGNOLI(2); SÍNTIA ZITZKE FISCHER ${ }^{(2)}$ e MÁRCIO PAIM MARIOT(2)
}

\begin{abstract}
RESUMO
Além da estética, o paisagismo contemporâneo visa a proporcionar diversos outros benefícios ao homem e ao meio ambiente, especialmente relacionados à qualidade ambiental dos espaços urbanos e à conservação das espécies. Uma das tendências nesse sentido é a redução no uso de plantas exóticas em seus projetos, visto que, com o tempo, podem se transformar em agentes de substituição da flora nativa, a exemplo do que ocorreu no Rio Grande do Sul com várias espécies introduzidas pelos colonizadores. O uso de espécies exóticas justifica-se cada vez menos, visto que a diversidade da flora do Bioma Pampa oferece expressiva quantidade de espécies nativas com características apropriadas para o uso ornamental. O cultivo comercial e a implantação de espécies nativas em áreas ajardinadas configuram inovações para viveiros e paisagistas, trazem ação positiva na redução do extrativismo, contribuem para a divulgação, valorização e preservação da flora nativa e reduzem o impacto que os insumos químicos promovem sobre o meio ambiente. Baseado no exposto, este trabalho teve por objetivo identificar espécies nativas do Bioma Pampa com características e usos semelhantes aos de espécies exóticas de uso consagrado no paisagismo brasileiro. As espécies foram selecionadas a partir da consulta a livros sobre plantas nativas do Bioma Pampa e plantas utilizadas no paisagismo brasileiro, por meio da semelhança de hábito, arquitetura e características das folhas, flores e/ou frutos, bem como das condições ambientais de ocorrência e de cultivo. Foram identificadas 34 espécies nativas capazes de substituir adequadamente espécies exóticas de uso consolidado. Os resultados evidenciam que existem diversas espécies nativas do Bioma Pampa que apresentam atributos ornamentais de interesse para o paisagismo, permitindo a substituição de espécies exóticas tradicionalmente cultivadas.
\end{abstract}

Palavras chave: ganhos ambientais, preservação da biodiversidade, Bioma Pampa

\begin{abstract}
Native species that can replace exotic species in landscaping

Beyond aesthetics, the contemporary landscaping intends to provide other benefits for humans and environment, especially related to the environmental quality of urban spaces and conservation of the species. A trend in this direction is the reduction in the use of exotic plants in their designs, since, over time, they can become agents of replacement of native flora, as it has occurred in Rio Grande do Sul with many species introduced by settlers. However, the use of exotic species is unjustifiable, because the flora diversity of the Bioma Pampa offers many native species with appropriate features to the ornamental use. The commercial cultivation and the implantation of native species in landscaped areas constitute innovations for plant nurseries and landscapers and can provide a positive reduction in extractivism, contributing to dissemination, exploitation and preservation of native flora, and also decrease the impact of chemical products on environment. So, this work intends to identify native species of Bioma Pampa with features and uses similar to the most used exotic species at Brazilian landscaping. The species were selected from consulting books about native plants of Bioma Pampa and plants used at Brazilian landscaping, considering the similarity on habit and architecture, as well as characteristics of leafs, flowers and/or fruits and environmental conditions of occurrence and cultivation. There were identified 34 native species able to properly replace exotic species commonly used. The results show that many native species of Bioma Pampa have interesting ornamental features to landscape gardening, allowing them to replace exotic species that are traditionally cultivated.
\end{abstract}

Keywords: environmental benefits, biodiversity preservation, Bioma Pampa

\section{INTRODUÇÃO}

Além de fonte de alimentos, fibras, madeiras e fármacos, as plantas tornam o ambiente mais agradável e proporcionam benefícios intangíveis ao homem, como a redução dos níveis de estresse, a tolerância à dor e o incremento da produtividade no trabalho (LOHR, 2011). Nesse sentido, o paisagismo pode ser utilizado como instrumento para aliar esses benefícios a outros adicionais, relacionados à qualidade ambiental urbana e à conservação das espécies. Gengo e Henkes (2013) observam que o equilíbrio ecológico das grandes cidades é cada vez mais dependente do paisagismo, em decorrência da crescente ocupação dos espaços verdes naturais. Por essa razão, além da estética, o paisagismo tem buscado a melhoria da qualidade ambiental dos espaços urbanos. Uma das medidas nessa direção é a tendência em reduzir ou evitar, na medida do possível, o uso de plantas exóticas em seus projetos. A maior parte das plantas ornamentais utilizadas no mundo não é nativa das regiões onde é cultivada, podendo trazer consequências negativas aos ambientes naturais ao se tornarem agentes de substituição da flora

\footnotetext{
(1) Trabalho recebido para publicação em 25/09/2014 e aprovado em 17/06/2015

(2) Instituto Federal Sul-Rio-grandense, Câmpus Pelotas-Visconde da Graça, Pelotas-RS, Brasil. *Autor correspondente: elisabethstumpf@cavg.ifsul.edu.br
} 
nativa com o tempo (HEIDEN e IGANCI, 2009a). Isso é observado no estado do Rio Grande do Sul, onde o arraigado costume de priorizar espécies exóticas em vez de nativas remonta à época da colonização, quando os imigrantes traziam consigo plantas para cultivar em seus novos jardins, como forma de reproduzir os ambientes de onde vieram (STUMPF et al., 2009a). Heiden e Iganci (2009a) relatam que algumas das plantas introduzidas no estado naquela época, como o asparguinho (Asparagus setaceus), a madressilva (Lonicera japonica), o cafezinho (Pittosporum undulatum) e o tojo (Ulex europaeus), dentre outras, se tornaram invasoras e hoje ocupam o espaço natural de espécies nativas.

Interessante ressaltar que o Pampa brasileiro, bioma restrito ao Rio Grande do Sul, abriga grande diversidade de vegetação em seus campos e matas, com expressiva quantidade de espécies nativas que evidenciam características para o uso ornamental (STUMPF et al., 2012). O reconhecimento desse potencial, com base na produção em escala comercial, configura possibilidades inovadoras para viveiros e paisagistas, alavancando, consequentemente, a cadeia produtiva da floricultura. A produção comercial de plantas nativas pode contribuir para a divulgação, valorização e preservação da biodiversidade e diminuição do extrativismo. Esses benefícios tornamse ainda mais relevantes se for levado em conta que, no Pampa, restam pouco mais de $40 \%$ de vegetação nativa em diferentes estados de conservação (HEIDEN e IGANCI, 2009b). Nessa perspectiva, Silva e Perelló (2010) defendem que a utilização de espécies nativas em espaços ajardinados pode atender a objetivos de educação ambiental, pela difusão da identidade paisagística natural e regional. Heiden et al. (2007), por sua vez, mencionam a oferta de ambientes para a avifauna local e a possibilidade de redução dos custos de manutenção e do uso de insumos químicos, pela adaptação edafo-climática das espécies, como outros resultados importantes que essa nova prática pode oferecer.

Fischer et al. (2007) alertam que, no Brasil, a falta de pesquisas sobre plantas ornamentais nativas leva à subutilização do potencial de sua flora. De fato, a oferta de espécies nativas em viveiros comerciais não é comum (LEAL e BIONDI, 2006), o que restringe, ou mesmo impede seu uso em projetos de paisagismo. As autoras citam que muitas espécies nativas, especialmente as que ocorrem em áreas ameaçadas pelo processo de urbanização, podem se extinguir antes de se tornarem conhecidas e terem seu potencial reconhecido e utilizado. Dessa forma, fica mais uma vez evidenciado que o cultivo comercial e o uso de espécies nativas em áreas ajardinadas são importantes instrumentos de conservação dessas espécies. No entanto, para que a adoção de espécies nativas no paisagismo tenha sucesso, é preciso reconhecer nelas características análogas às das exóticas de uso consolidado. Desse modo, além de características morfológicas semelhantes, é preciso que elas se adaptem às mesmas condições ambientais e atendam a iguais funções dentro do cenário pretendido.

Considerando a deficiência de informações sobre o potencial de uso da flora nativa do Bioma Pampa no paisagismo, e com base no cenário apresentado, este trabalho tem por objetivo identificar espécies nativas do Pampa com características e potencial de uso semelhantes aos de espécies exóticas de uso consagrado no paisagismo brasileiro. A apresentação dessas espécies servirá para subsidiar pesquisas de cultivo e adaptação, para futura implantação em espaços ajardinados, substituindo espécies exóticas análogas.

\section{MATERIAL E MÉTODOS}

A fim de identificar espécies nativas que apresentassem analogias com espécies exóticas, o trabalho analisou e comparou a descrição e imagens das espécies contidas no livro Cores e Formas no Bioma Pampa: plantas ornamentais nativas (STUMPF et al., 2009b), que apresenta 100 espécies nativas com potencial ornamental ainda não cultivadas comercialmente e o livro Plantas Ornamentais do Brasil: arbustivas, herbáceas e trepadeiras (LORENZI e SOUZA, 2008), que mostra as principais espécies cultivadas em jardins. A seleção das espécies levou em conta a semelhança de hábito, de arquitetura e características das folhas, flores e/ou frutos, bem como as condições ambientais de ocorrência (para as espécies nativas) e de cultivo (para as espécies exóticas).

\section{RESULTADOS E DISCUSSÃO}

A partir da consulta bibliográfica, foram identificadas 34 espécies nativas do Bioma Pampa (Figura 1) com características análogas a 34 espécies exóticas cultivadas em jardins do Brasil.

Ressalta-se que algumas espécies nativas podem substituir mais de uma espécie exótica (como Hibiscus diversifolius substituindo as exóticas Hibiscus acetosella e $H$. rosa-sinensis) e, por outro lado, algumas exóticas podem ser substituídas por mais de uma espécie nativa (Helychrisum petiolatum por Baccharis ochracea, Croton quintasii e Lucilia acutifolia, por exemplo), como pode ser observado na Tabela 1 . 

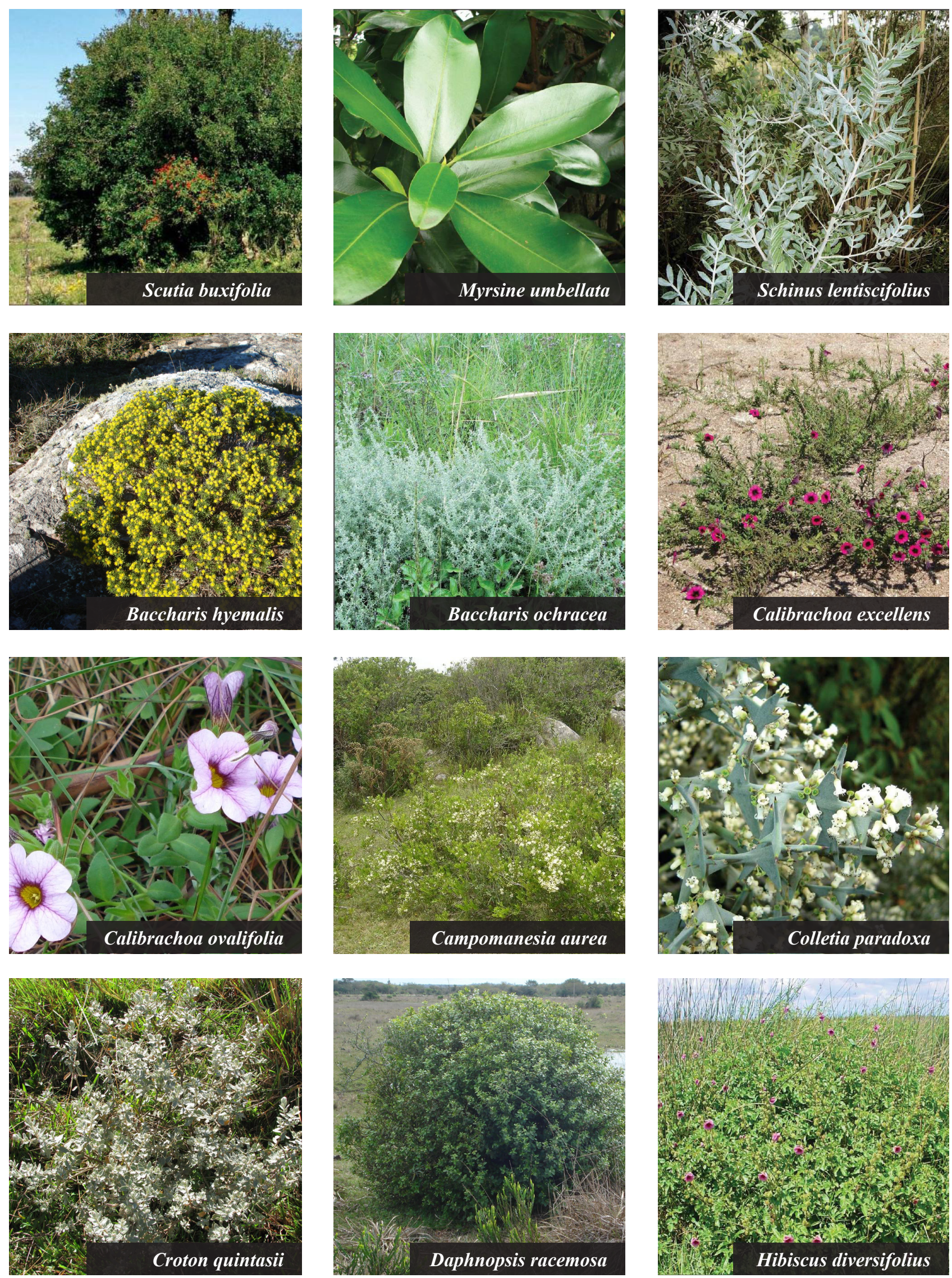

Figura 1. Espécies nativas do Bioma Pampa com características análogas as de espécies exóticas tradicionalmente utilizadas no paisagismo. (Continua).

Figure 1. Native species of the Pampa biome with characteristics similar to those of exotic species traditionally used in landscaping. (To be continued). 

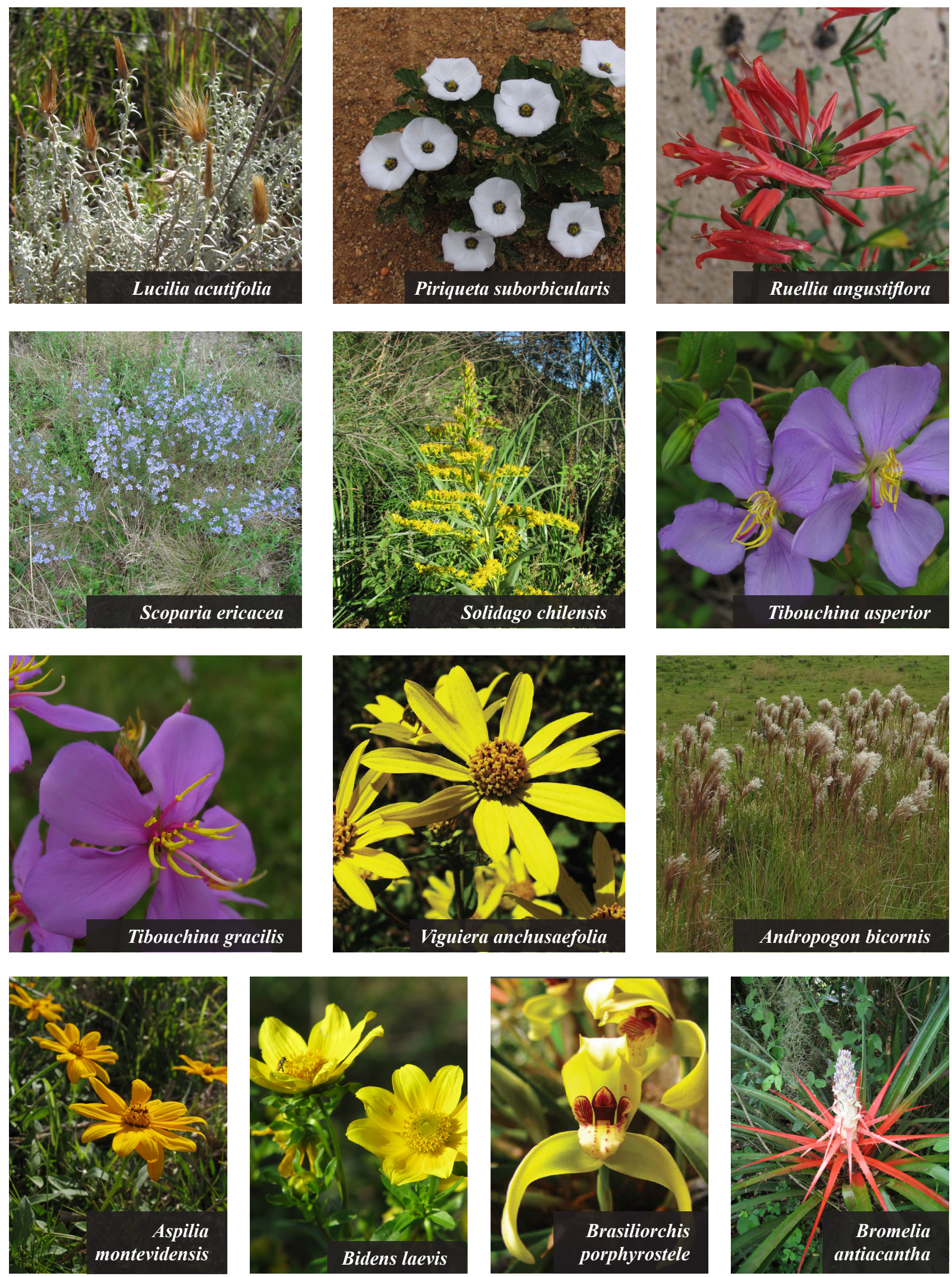

Figura 1. Espécies nativas do Bioma Pampa com características análogas as de espécies exóticas tradicionalmente utilizadas no paisagismo. (Continua).

Figure 1. Native species of the Pampa biome with characteristics similar to those of exotic species traditionally used in landscaping. (To be continued). 

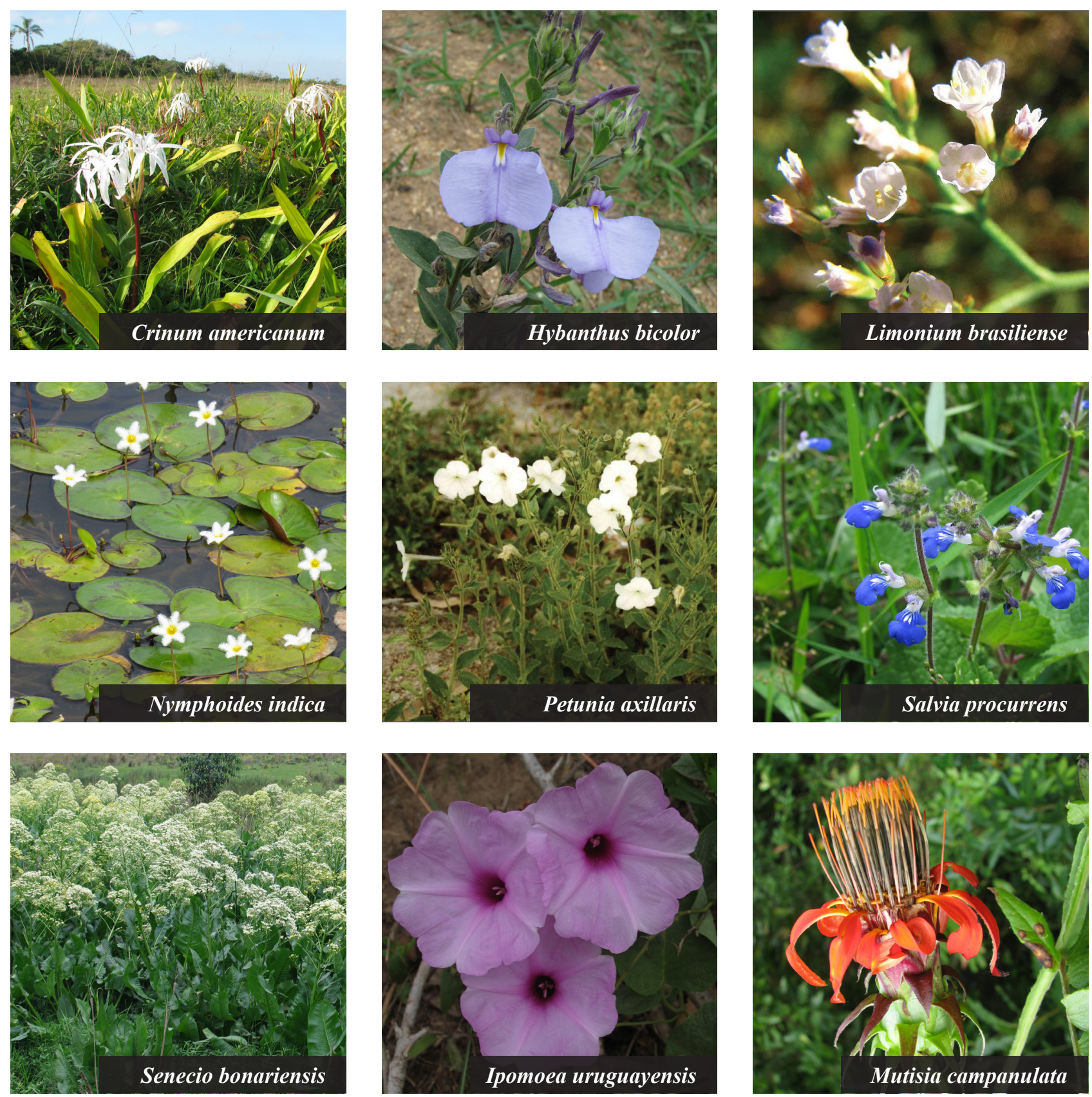

Figura 1. Espécies nativas do Bioma Pampa com características análogas as de espécies exóticas tradicionalmente utilizadas no paisagismo. (Conclusão).

Figure 1. Native species of the Pampa biome with characteristics similar to those of exotic species traditionally used in landscaping. (Conclusion). 
Tabela 1. Espécies nativas do Bioma Pampa com características análogas as de espécies exóticas tradicionalmente utilizadas no paisagismo.

Table 1. Native species of the Pampa biome with characteristics similar to those of exotic species traditionally used in landscaping.

\begin{tabular}{|c|c|c|c|}
\hline НÁBITO & ESPÉCIE NATIVA & ESPÉCIE EXÓTICA & $\begin{array}{c}\text { CARACTERÍSTICAS } \\
\text { SEMELHANTES ENTRE AS } \\
\text { ESPÉCIES }\end{array}$ \\
\hline \multirow{3}{*}{ ÁRVORES } & Scutia buxifolia & Pyracantha coccinea & arquitetura, espinhos guardiães, frutos \\
\hline & Myrsine umbellata & Schefflera actinophylla & arquitetura, cor e textura de folhas \\
\hline & Schinus lentiscifolius & Eucalyptus cinerea & cor de folhas \\
\hline \multirow{17}{*}{$\begin{array}{c}\text { ARBUSTOS } \\
\text { E } \\
\text { SUBARBUSTOS }\end{array}$} & Baccharis hyemalis & Thuja orientalis rosedallis-aurea & $\begin{array}{l}\text { arquitetura, coloração na época da } \\
\text { floração de } B \text {. hyemalis }\end{array}$ \\
\hline & Baccharis ochracea & $\begin{array}{l}\text { Centaurea gymnocarpa } \\
\text { Helychrisum petiolatum } \\
\text { Senecio douglasii }\end{array}$ & arquitetura, cor de folhas \\
\hline & Calibrachoa excellens & Petunia $x$ hybrida & arquitetura, cor de flores \\
\hline & Calibrachoa ovalifolia & Petunia $x$ hybrida & arquitetura, cor de flores \\
\hline & Campomanesia aurea & Viburnum tinus & arquitetura, cor de flores \\
\hline & Colletia paradoxa & Euphorbia milli & espinhos guardiães \\
\hline & Croton quintasii & $\begin{array}{l}\text { Centaurea gymnocarpa } \\
\text { Helychrisum petiolatum } \\
\text { Senecio douglasii }\end{array}$ & arquitetura, cor de folhas \\
\hline & Daphnopsis racemosa & Buxus sempervirens & arquitetura, cor de folhas \\
\hline & Hibiscus diversifolius & $\begin{array}{l}\text { Hibiscus acetosella } \\
\text { Hibiscus rosa-sinensis }\end{array}$ & arquitetura, cor de flores \\
\hline & Lucilia acutifolia & $\begin{array}{l}\text { Centaurea gymnocarpa } \\
\text { Helychrisum petiolatum } \\
\text { Santolina chamaecyparis }\end{array}$ & cor de folhas \\
\hline & Piriqueta suborbicularis & Turnera subulata & arquitetura, cor de flores \\
\hline & Ruellia angustiflora & Odontonema tubaeforme & arquitetura, cor de flores \\
\hline & Scoparia ericacea & $\begin{array}{l}\text { Cuphea gracilis } \\
\text { Felicia amelloides }\end{array}$ & arquitetura, cor de flores \\
\hline & Solidago chilensis & Solidago canariensis & arquitetura, cor de flores \\
\hline & Tibouchina asperior & Solanum rantonnetii & arquitetura, cor de flores \\
\hline & Tibouchina gracilis & Solanum rantonnetii & arquitetura, cor de flores \\
\hline & Viguiera anchusaefolia & Argyranthemum frutescens & arquitetura, cor de flores \\
\hline \multirow{12}{*}{ HERBÁCEAS } & Andropogon bicornis & Pennisetum setaceum & arquitetura, cor de flores \\
\hline & Aspilia montevidensis & Bidens rubifolia & arquitetura, cor de flores \\
\hline & Bidens laevis & Bidens rubifolia & arquitetura, cor de flores \\
\hline & $\begin{array}{l}\text { Brasiliorchis } \\
\text { porphyrostele }\end{array}$ & Cymbidium $x$ hybridum & arquitetura, cor de flores \\
\hline & Bromelia antiacantha & Guzmania sanguinea & arquitetura, cor de folhas \\
\hline & Crinum americanum & Hedychium coronarium & arquitetura, cor de flores \\
\hline & Hybanthus bicolor & Browallia americana & arquitetura, cor de flores \\
\hline & Limonium brasiliense & Limonium latifolia & arquitetura, cor de flores \\
\hline & Nymphoides indica & Nymphaea alba & arquitetura, cor de flores \\
\hline & Petunia axillaris & Petunia $x$ hybrida & arquitetura, cor de flores \\
\hline & Salvia procurrens & $\begin{array}{c}\text { Vinca major } \\
\text { Pilea nummulariifolia }\end{array}$ & $\begin{array}{l}\text { arquitetura, cor de flores } \\
\text { arquitetura, cor de folhas }\end{array}$ \\
\hline & Senecio bonariensis & Hedychium coronarium & arquitetura, cor de flores \\
\hline \multirow{2}{*}{ TREPADEIRAS } & Ipomoea uruguayensis & Argyreia nervosa & arquitetura, cor de flores \\
\hline & Mutisia campanulata & Pseudogynoxys chenopodioides & arquitetura, cor de flores \\
\hline
\end{tabular}


Muitas espécies nativas do Pampa apresentaram semelhanças com as espécies exóticas cultivadas em jardins no tocante à arquitetura de planta e cor de flores. Scutia buxifolia e Colletia paradoxa foram destacadas pela presença e utilidade de seus espinhos e seis outras espécies apresentaram, como destaque, a semelhança na cor de folhas. Isso não significa, no entanto, que essas características sejam as únicas que justificam a substituição das espécies exóticas análogas, pois todas as nativas apresentam, também, outros atributos que justificariam plenamente sua implantação em áreas ajardinadas. Scutia buxifolia e Myrsine umbellata são árvores que apresentam frutos atrativos para a avifauna (STUMPF et al., 2009b) e podem ser cultivadas em parques, praças e demais áreas com grandes espaços disponíveis. No entanto, pela altura que atingem, não são indicadas para locais que possuam redes suspensas de eletricidade ou telefonia. Pela presença de espinhos nos ramos, Scutia buxifolia pode ser cultivada como planta guardiã em locais com pouca circulação de pessoas ou animais.

Pelas características apresentadas na Tabela 1 , as espécies nativas de porte arbustivo e subarbustivo podem substituir as exóticas na implantação de cercas-vivas (algumas sem necessidade de podas de conformação, como Baccharis hyemalis e Daphnopsis racemosa) e também para compor maciços ou forrações. Colletia paradoxa, espécie nativa com espinhos, pode ser empregada como planta-guardiã, compondo cercas-vivas de proteção. Merece destaque também a atração que as flores de Ruellia angustiflora exercem sobre beija-flores (STUMPF et al., 2009b). A produção e o cultivo de Colletia paradoxa e Tibouchina asperior, em especial, terá ação direta sobre sua conservação, visto que constam como vulnerável e em perigo, respectivamente, na Lista de Espécies da Flora Nativa Ameaçadas de Extinção no Estado do Rio Grande do Sul (RIO GRANDE DO SUL, 2002).

As espécies herbáceas nativas, por sua vez, podem substituir as espécies exóticas análogas no cultivo em canteiros localizados a pleno sol (Andropogon bicornis, Bidens laevis, Hybanthus bicolor e Petunia axillaris, por exemplo), a meia-sombra (como Salvia procurrens) e até mesmo em áreas alagadiças (Crinum americanum, Limonium brasiliense, Nymphoides indica e Senecio bonariensis).

Das espécies trepadeiras nativas selecionadas, destacase a dupla finalidade de Ipomoea uruguayensis, que pode ser cultivada também como planta rasteira, compondo forrações em conjunto com outras espécies, e a atração que as flores de Mutisia campanulata exercem sobre beijaflores.

\section{CONCLUSÃO}

Os resultados obtidos evidenciam que existem diversas espécies nativas do Bioma Pampa que apresentam atributos ornamentais de interesse para o paisagismo, permitindo a substituição de espécies exóticas tradicionalmente cultivadas.

\section{AGRADECIMENTOS}

Ao CNPq, pela concessão das bolsas de iniciação científica.

\section{REFERÊNCIAS}

FISCHER, S.Z.; STUMPF, E.R.T.; HEIDEN, G.; BARBIERI, R.L.; WASUM, R.A. Plantas da flora brasileira no mercado internacional de floricultura. Revista Brasileira de Biociências, Porto Alegre, v.5, p.510-512, 2007.

GENGO, R.C.; HENKES, J.A. A utilização do paisagismo como ferramenta na preservação e melhoria ambiental em área urbana. Revista Gestão \& Sustentabildade Ambiental, Florianópolis, v.1, n.2, p.55-81, 2013.

HEIDEN, G.; STUMPF, E.R.T.; BARBIERI, R.L.; GROLLI, P.R. Uso de plantas subarbustivas e herbáceas nativas do Rio Grande do Sul como alternativa a ornamentais exóticas. Revista Brasileira de Agroecologia, Porto Alegre, v.2. p.850-853, 2007.

HEIDEN, G.; IGANCI, J.R.V. Valorizando a flora nativa. In: STUMPF, E.R.T.; BARBIERI, R.L.; HEIDEN, G. Cores e formas no Bioma Pampa: plantas ornamentais nativas. Pelotas: Embrapa Clima Temperado, 2009a. p.36-43.

HEIDEN, G.; IGANCI, J.R.V. Sobre a paisagem e a flora. In: STUMPF, E.R.T.; BARBIERI, R. L.; HEIDEN, G. (Org.). Cores e formas no Bioma Pampa: plantas ornamentais nativas. Pelotas: Embrapa Clima Temperado, 2009b. p.22-35.

LEAL, L.; BIONDI, D. Potencial ornamental de espécies nativas. Revista Científica Eletrônica de Engenharia Florestal, Garça, v.4, n.8, p.1-16, 2006.

LOHR, V. Greening the human environment: the untold benefits. Acta Horticulturae, Lisboa, v.916, p.159-170, 2011.

LORENZI, H.; SOUZA, H.M. Plantas ornamentais no Brasil: arbustivas, herbáceas e trepadeiras. Nova Odessa: Instituto Plantarum, 2008. 1088 p.

RIO GRANDE DO SUL. Assembleia Legislativa do Estado do Rio Grande do Sul. Decreto no 42.099/2002. Declara as espécies da flora nativa ameaçadas de extinção no Estado do Rio Grande do Sul e dá outras providências. Disponível em: <http://www.al.rs.gov.br/ legis/M010/M0100099.ASP?Hid_Tipo=TEXTO\&Hid TodasNormas $=320 \&$ hTexto $=\&$ Hid_IDNorma $=320>$. Acesso em: 18 de setembro de 2014. 
SILVA, J. G.; PERELLÓ, L.F.C. Conservação de espécies ameaçadas do Rio Grande do Sul através de seu uso no paisagismo. Revista da Sociedade Brasileira de Arborização Urbana, Piracicaba, v.5, n.4, p.1-21, 2010.

STUMPF E.R.T.; ROMANO, C.M.; BARBIERI, R.L.; HEIDEN, G.; FISCHER, S.Z.; CORREAA, L.B. Características ornamentais de plantas do Bioma Pampa. Revista Brasileira de Horticultura Ornamental, v.15, n.1, p.49-62, 2009a.
STUMPF, E.R.T.; BARBIERI, R.L.; HEIDEN, G. (Org.). Cores e Formas no Bioma Pampa: plantas ornamentais nativas. Pelotas: Embrapa Clima Temperado, 2009b. 276 p.

STUMPF, E.R.T.; HEIDEN, G.; IGANCI, J.R.V.; BARBIERI, R.L.; CORRÊA, L.B.; PERLEBERG, T.D.; ROMANO, C.M.; FISCHER, S.Z.; NEITZKE, R.S. Prospecting native ornamental plants in the Brazilian pampa for use in landscaping and floral art. Acta Horticulturae, Lisboa, v.937, p.1161-1166, 2012. 